\title{
Single-vesicle imaging quantifies calcium's regulation of nanoscale vesicle clustering mediated by a-synuclein
}

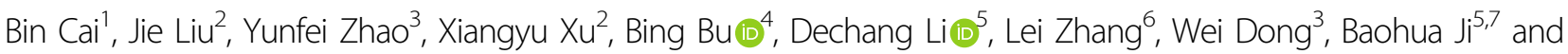 \\ Jiajie Diao (1) ${ }^{1}$
}

\begin{abstract}
Although numerous studies have shown that the protein a-synuclein (a-Syn) plays a central role in Parkinson's disease, dementia with Lewy bodies, and other neurodegenerative diseases, the protein's physiological function remains poorly understood. Furthermore, despite recent reports suggesting that, under the influence of $\mathrm{Ca}^{2+}$, a-Syn can interact with synaptic vesicles, the mechanisms underlying that interaction are far from clear. Thus, we used singlevesicle imaging to quantify the extent to which $\mathrm{Ca}^{2+}$ regulates nanoscale vesicle clustering mediated by a-Syn. Our results revealed not only that vesicle clustering required a-Syn to bind to anionic lipid vesicles, but also that different concentrations of $\mathrm{Ca}^{2+}$ exerted different effects on how a-Syn induced vesicle clustering. In particular, low concentrations of $\mathrm{Ca}^{2+}$ inhibited vesicle clustering by blocking the electrostatic interaction between the lipid membrane and the $\mathrm{N}$ terminus of a-Syn, whereas high concentrations promoted vesicle clustering, possibly due to the electrostatic interaction between $\mathrm{Ca}^{2+}$ and the negatively charged lipids that is independent of a-Syn. Taken together, our results provide critical insights into a-Syn's physiological function, and how $\mathrm{Ca}^{2+}$ regulates vesicle clustering mediated by a-Syn.
\end{abstract}

\section{Introduction}

$\alpha$-Synuclein ( $\alpha$-Syn), a presynaptic protein abundantly expressed throughout the central nervous system, is the hallmark of Parkinson's disease, dementia with Lewy bodies, and other neurodegenerative diseases ${ }^{1,2}$. Found to aggregate in a nucleation-dependent manner, $\alpha$-Syn forms cytotoxic amyloid oligomers and fibrils ${ }^{3-8}$. Recent studies have indicated that $\alpha$-Syn not only binds to the highly curved membranes of synaptic vesicles, but also senses and regulates the curvature of those membranes, which

\footnotetext{
Correspondence: Dechang Li (dcli@zju.edu.cn) or Lei Zhang (zhangleio@xjtu. edu.cn) or Wei Dong (dongwei@swmu.edu.cn) or Jiajie Diao (jiajie.diao@uc.edu)

'Department of Cancer Biology, University of Cincinnati College of Medicine, Cincinnati, $\mathrm{OH} 45267$, USA

${ }^{2}$ Biomechanics and Biomaterials Laboratory, Department of Applied Mechanics, Beijing Institute of Technology, Beijing 100081, China Full list of author information is available at the end of the article These authors contributed equally: Bin Cai, Jie Liu, Yunfei Zhao
}

immediately suggests that $\alpha$-Syn is involved in synaptic vesicle trafficking and its exo- and endocytosis ${ }^{9-11}$. However, the physiological function of monomeric $\alpha$-Syn remains unclear ${ }^{9,12}$.

In aqueous solution, $\alpha$-Syn is an intrinsically disordered protein that binds to small synaptic vesicles via a conserved lipid-binding domain. When binding to a lipid membrane, the $\mathrm{N}$ terminus of $\alpha$-Syn forms either an extended helix structure or two broken structures ${ }^{13,14}$. It has also been proposed that $\alpha$-Syn acts as a bridge between membranes-for example, that two helices each bind to two different synaptic vesicles-the result of which is vesicle clustering ${ }^{15}$. At the same time, recent studies have suggested that calcium ions $\left(\mathrm{Ca}^{2+}\right)$ can mediate the interaction between $\alpha$-Syn and lipid membranes. For instance, Zhang et al. demonstrated that $\mathrm{Ca}^{2+}$ competitively binds to anionic lipids, which triggers the dissociation of $\alpha$-Syn from membranes ${ }^{16,17}$. However,

\section{(c) The Author(s) 2020}

(c) (i) Open Access This article is licensed under a Creative Commons Attribution 4.0 International License, which permits use, sharing, adaptation, distribution and reproduction cc) in any medium or format, as long as you give appropriate credit to the original author(s) and the source, provide a link to the Creative Commons license, and indicate if changes were made. The images or other third party material in this article are included in the article's Creative Commons license, unless indicated otherwise in a credit line to the material. If material is not included in the article's Creative Commons license and your intended use is not permitted by statutory regulation or exceeds the permitted use, you will need to obtain permission directly from the copyright holder. To view a copy of this license, visit http://creativecommons.org/licenses/by/4.0/. 
upon finding that $\mathrm{Ca}^{2+}$ increases $\alpha$-Syn's binding to anionic lipids via $\alpha$-Syn's $C$ terminus, Lautenschläger et $\mathrm{al}^{18}$. proposed that the neutralization of negative charges on residues at the $\mathrm{C}$ terminus via the dynamic binding of positively charged $\mathrm{Ca}^{2+}$ facilitates $\alpha$-Syn's interaction with phospholipid membranes. They also showed that $\alpha$-Syn and $\mathrm{Ca}^{2+}$ balance the electrostatic interaction of synaptic vesicles, which facilitates vesicle clustering $^{18}$. Another recent study revealed that $\alpha$-Syn amyloid oligomers, acting as multivalent nanoparticles, can cause hemifusion in negatively charged vesicles ${ }^{19}$. However, how $\mathrm{Ca}^{2+}$ regulates the interaction of $\alpha$-Syn and lipids remains uncertain.

Currently, researchers are in great need of a technique for studying the interactions between $\alpha$-Syn and lipid membranes at the single-particle level ${ }^{20}$. As one option, total internal reflection fluorescence microscopy (TIRFM) is an optical technique for inducing an evanescent wave or field in a limited region of a specimen immediately adjacent to the interface between two media with different refractive indices ${ }^{21,22}$. A typical setup for TIRFM is shown in Fig. 1. Because the evanescent wave decays exponentially with distance from the interface's surface, only fluorescent molecules within a few hundred nanometers of the interface are excited efficiently. In this way, TIRFM facilitates the excitation and observation of fluorophores within an extremely limited axial region ${ }^{21,22}$. Thus, TIRFM has benefited studies requiring images of individual particles in specimens with large numbers of fluorophores outside the optical plane of interest ${ }^{21,22}$, including during vesicle docking induced by proteins ${ }^{23-26}$.

In our study, we used single-vesicle imaging to quantify the extent to which $\mathrm{Ca}^{2+}$ regulates vesicle clustering mediated by $\alpha$-Syn and to investigate the possible underlying mechanism. Among our results, $\alpha$-Syn induced clustering of anionic lipid vesicles when no $\mathrm{Ca}^{2+}$ interaction occurred. Moreover, vesicle clustering induced by $\alpha$-Syn was inhibited in the presence of low concentrations of $\mathrm{Ca}^{2+}$, where competitive binding of $\mathrm{Ca}^{2+}$ to lipids altered the electrostatic interaction and, in turn, caused the $\mathrm{N}$ terminus of $\alpha$-Syn to dissociate from the membranes. By contrast, although $\mathrm{Ca}^{2+}$ inhibited the interaction of $\alpha$-Syn and the lipid membranes, a high concentration of $\mathrm{Ca}^{2+}$ supplied sufficient multivalent ions to bridge vesicles into close contact and enhance vesicle clustering. Our results provide critical insights into $\alpha$ Syn's physiological function and the mechanism by which calcium ions regulate vesicle clustering mediated by $\alpha-$ Syn.

\section{Results \\ $\mathrm{Ca}^{2+}$ regulates vesicle clustering mediated by a-Syn depending on $\mathrm{Ca}^{2+}$ concentration}

To illustrate how $\mathrm{Ca}^{2+}$ regulates vesicle clustering with $\alpha$-Syn, we used transmission electron microscopy (TEM) to image lipid vesicles with $\alpha$-Syn in the absence of $\mathrm{Ca}^{2+}$. Figure 2 shows representative TEM images of $\alpha$-Syn with lipid vesicles composed of 1,2-dioleoyl-sn-glycero-3phosphocholine (DOPC) only or DOPC plus 1,2-dioleoylsn-glycero-3-phospho-L-serine (DOPS). As shown in Fig. $2 a$, because $\alpha$-Syn rarely binds to vesicles without anionic lipids, the $100 \%$ DOPC vesicles dispersed in the solution. By contrast, numerous DOPS:DOPC (12\%:88\%) vesicles formed clusters with $\alpha$-Syn, which indicates that vesicle clustering requires $\alpha$-Syn to bind to anionic lipid vesicles, as shown in Fig. 2b. However, TEM measurements cannot quantify the capability of vesicle clustering induced by $\alpha$-Syn.

To quantify vesicle clustering induced by $\alpha$-Syn, we performed a single-vesicle assay with two types of fluorescent dye to label different vesicles-free vesicles containing the fluorescent molecule DiI (DiIC18(3)) and

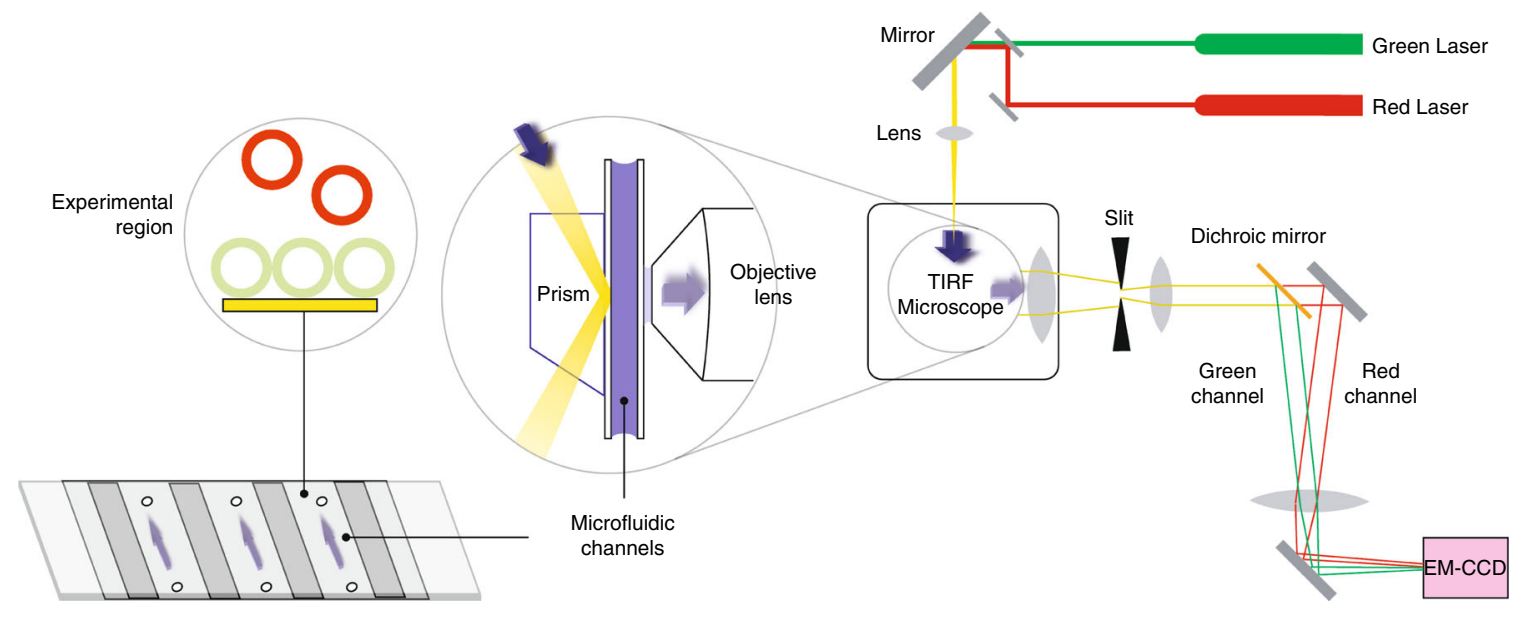

Fig. 1 Illustration of the TIRFM system for studying the clustering of vesicles 

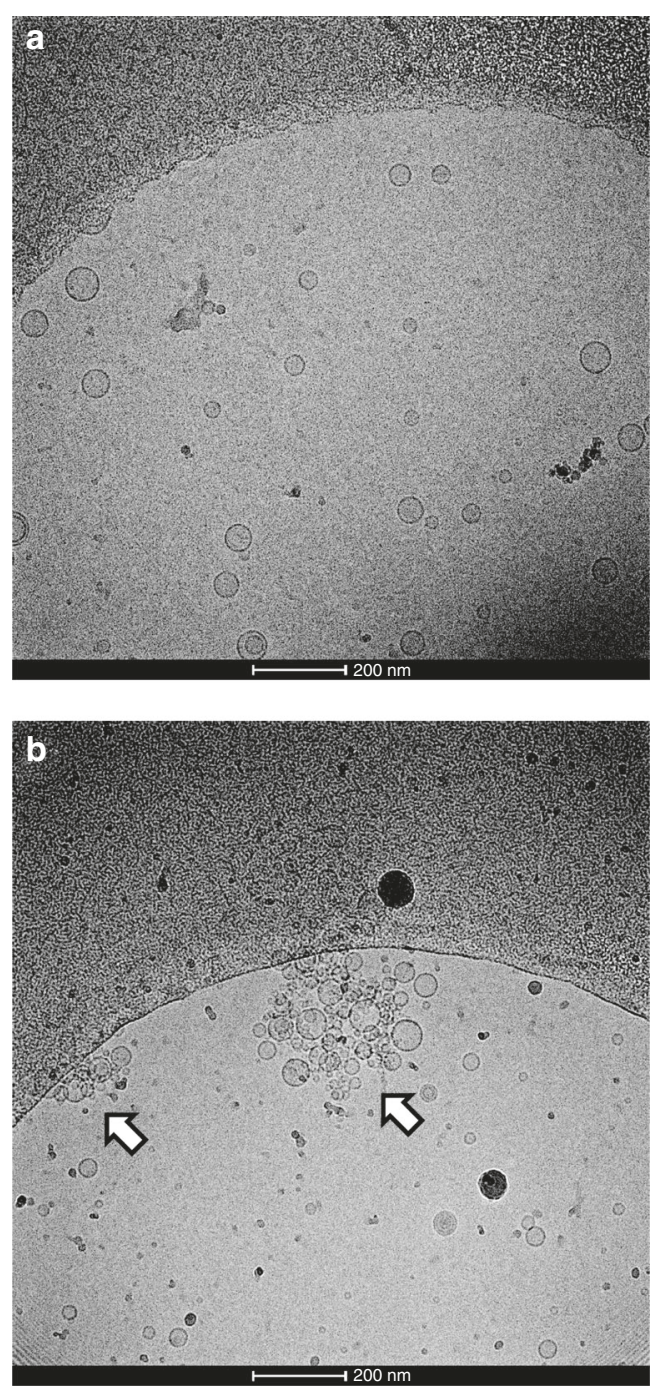

Fig. 2 a-Syn induces liposome clustering. Representative TEM images of a-Syn with a DOPC vesicles and $\mathbf{b} 12 \%$ DOPS:DOPC $(12 \%: 88 \%)$ vesicles. The arrows indicate the vesicle clusters. The protein-to-lipid ratio was 1:500. The incubation time was $60 \mathrm{~min}$

immobilized vesicles containing the fluorescent molecule DiD (DiIC18(5)). The detection of vesicle clustering relied upon TIRFM (Fig. 3a), which can reveal individual vesicles clustered on the surface of immobilized vesicles. As shown in Fig. 3b, c, the concentrations of $\mathrm{Ca}^{2+}$ exerted a significant effect on vesicle clustering. At concentrations of $0.5 \mathrm{mM}$ and $2 \mathrm{mM} \mathrm{Ca}{ }^{2+}$, there were fewer vesicle clusters than in the control without $\mathrm{Ca}^{2+}$, which indicates that $\mathrm{Ca}^{2+}$ at concentrations of $0.5 \mathrm{mM}$ and $2 \mathrm{mM}$ inhibits the interaction of $\alpha$-Syn with vesicles, as depicted in Fig. $3 b, c$. However, clusters at a concentration of $10 \mathrm{mM} \mathrm{Ca}^{2+}$ outnumbered those in the control, which suggests that higher concentrations of $\mathrm{Ca}^{2+}$ promote vesicle-vesicle interaction mediated by $\alpha$-Syn. Due to surface variation, the baseline level of vesicle clustering differed significantly across different imaging surfaces; therefore, only experiments on the same imaging slide are directly comparable with absolute counts. To compare the results from different slides, we calculated the normalized value of the clustering counts (Fig. 3c) based on the original counts (Fig. 3b). A normalized value above or below 1 indicated the promotional or inhibitory effect of $\mathrm{Ca}^{2+}$, respectively. The normalized results shown in Fig. 3c reveal the concentration-dependent effect of $\mathrm{Ca}^{2+}$ on vesicle clustering with $\alpha$-Syn, such that lower concentrations (i.e., 0.5 and $2 \mathrm{mM}$ ) of $\mathrm{Ca}^{2+}$ inhibited clustering, whereas a higher concentration (i.e., $10 \mathrm{mM}$ ) promoted it.

To cross-check our results, we performed a bulk vesicleclustering experiment in the presence of $\alpha$-Syn and different concentrations of $\mathrm{Ca}^{2+}$. We incubated $\alpha$-Syn with vesicles with $12 \%$ PS that were approximately $50 \mathrm{~nm}$ in diameter, and that had been labeled with the fluorescent molecule $\mathrm{DiD}$. Although no significant aggregates appeared in the solution with vesicles only (Fig. 4a), vesicles in the $\alpha$-Syn-containing solution formed some large aggregates, which confirmed that vesicle clustering is mediated by $\alpha-S y n$, as shown in Fig. 4b. However, the level of aggregation was reduced when $0.5 \mathrm{mM} \mathrm{Ca}^{2+}$ was introduced (Fig. 4c), which demonstrates the inhibitory effect of $0.5 \mathrm{mM} \mathrm{Ca}^{2+}$ on vesicle clustering. After incubation of vesicles in $10 \mathrm{mM} \mathrm{Ca}^{2+}$ in the presence of $\alpha$-Syn, an increasing number and size of aggregates formed, which shows that vesicle clustering is promoted by $10 \mathrm{mM} \mathrm{Ca}^{2+}$ (Fig. 4d). Such results confirmed the concentration-dependent effect of $\mathrm{Ca}^{2+}$ on vesicle clustering with $\alpha$-Syn, such that lower concentrations (i.e., 0.5 and $2 \mathrm{mM}$ ) of $\mathrm{Ca}^{2+}$ inhibited clustering, whereas a higher concentration (i.e., $10 \mathrm{mM}$ ) promoted clustering.

\section{$\mathrm{Ca}^{2+}$ regulates vesicle clustering via its electrostatic interaction with lipid membranes}

To gain insights into the mechanism by which $\mathrm{Ca}^{2+}$ regulates vesicle clustering, we performed vesicleclustering experiments in the presence and absence of $\alpha$-Syn with different concentrations of $\mathrm{Ca}^{2+}$. Moreover, to investigate whether electrically charged lipids affect the clustering interaction in the presence or absence of $\alpha$-Syn, we prepared lipid vesicles with no electric charge (i.e., no PS) as a control group. Figure 5a shows the clustering counts of vesicles with no PS and with $12 \%$ PS in a low concentration of $\mathrm{Ca}^{2+}$ (i.e., $0.5 \mathrm{mM}$ ) with and without $\alpha$ Syn. When $\alpha$-Syn was absent, no significant difference in vesicle-clustering counts was observed between the vesicles without PS and those with $12 \%$ PS, which indicates that low concentrations of $\mathrm{Ca}^{2+}$ do not affect vesicle clustering in the absence of $\alpha$-Syn. However, when $\alpha$-Syn was present, clustering counts in vesicles with $12 \%$ PS were significantly less than those in vesicles without PS. Thus, the result shown in Fig. 5a suggests that vesicle 
a

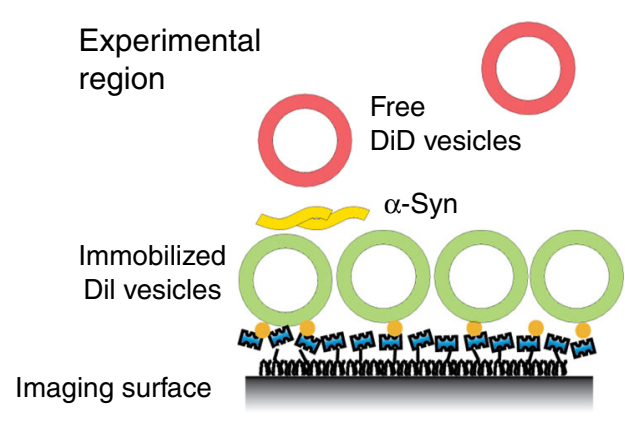

b

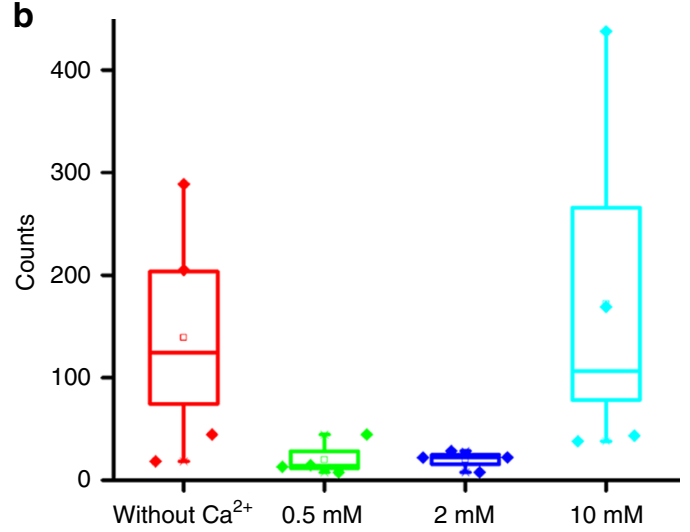

C
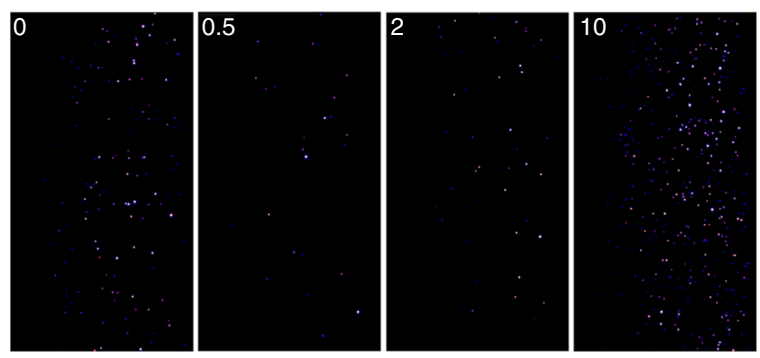

d

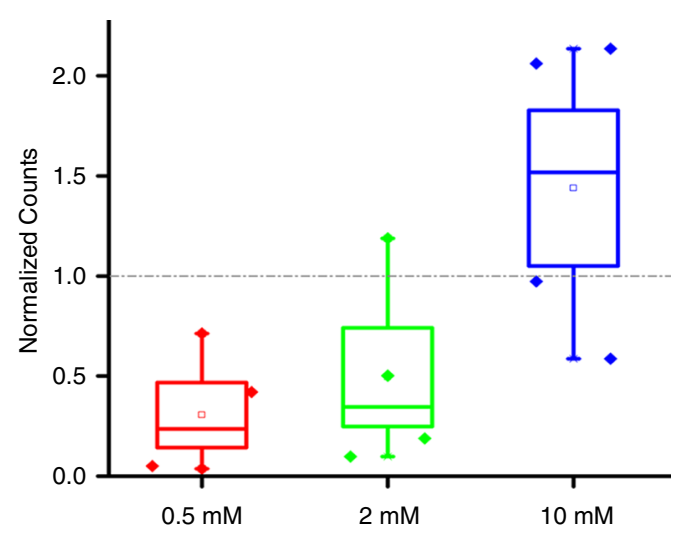

Fig. 3 A single-vesicle clustering assay reveals the effect of various $\mathrm{Ca}^{2+}$ concentrations on the vesicle-clustering counts. a Illustration of the single-vesicle clustering assay. $\mathbf{b}$ The clustering counts. c The representative TIRF images of each channel, and $\mathbf{d}$ the normalized results of $12 \%$ PS vesicle clustering regulated by a-Syn. The case without $\mathrm{Ca}^{2+}$ was used as the control result

clustering is mediated by $\alpha$-Syn and inhibited by $\mathrm{Ca}^{2+}$ at a low concentration (i.e., $0.5 \mathrm{mM}$ ).

By contrast, at a high concentration of $\mathrm{Ca}^{2+}$ (i.e., $10 \mathrm{mM}$ ) without $\alpha$-Syn, $12 \%$ PS increased the vesicleclustering counts, regardless of whether $\alpha$-Syn participated in the clustering process (Fig. 5b). The result suggests that $\mathrm{Ca}^{2+}$ can enhance vesicle clustering only at high concentrations with negatively charged lipids. The promotional role of $\mathrm{Ca}^{2+}$ at high concentrations (e.g., $10 \mathrm{mM}$ ) in vesicle clustering may occur via electrostatic interactions between $\mathrm{Ca}^{2+}$ and negatively charged lipids, as shown by earlier findings that multivalent ions may prompt ion bridges and tight coupling between lipid membranes $^{27}$. Thus, whereas a high concentration of $\mathrm{Ca}^{2+}$ causes many $\mathrm{Ca}^{2+}$ to bind to lipids, and bound multivalent ions can bridge vesicles to form clusters, a low concentration of $\mathrm{Ca}^{2+}$ cannot supply sufficient $\mathrm{Ca}^{2+}$ to drive vesicles into close contact.

\section{$\mathrm{Ca}^{2+}$ inhibits the interaction between $\mathrm{a}-\mathrm{Syn}$ and lipid membranes}

Having observed the inhibitory effect of low concentrations of $\mathrm{Ca}^{2+}$ and the promotional effect of high concentrations, we recorded the circular dichroism (CD) spectra of $\alpha$-Syn in CD buffer $\left(20 \mathrm{mM}\right.$ of $\mathrm{Na}_{2} \mathrm{HPO}_{4} /$
$\mathrm{NaH}_{2} \mathrm{PO}_{4}$ and $100 \mathrm{mM}$ of $\mathrm{NaF}, \mathrm{pH} 7.4$ ), as shown in Fig. 6. The spectrum of the control group (i.e., free $\alpha$-Syn only) had a minimum ellipticity of approximately $198 \mathrm{~nm}$, which suggests that free $\alpha$-Syn possesses a high percentage of random coil structures. After $\alpha$-Syn was mixed with lipid vesicles containing $12 \%$ PS, two new characteristic minima appeared at $210-222 \mathrm{~nm}$ without any inverse peak in the vicinity of $198 \mathrm{~nm}$, which demonstrates that highly unstructured $\alpha$-Syn undergoes a conformational change into a typical secondary structure (i.e., $\alpha$-helix) thought to be the stable conformation of lipidbound $\alpha$-Syn. Nevertheless, the intensity of the characteristic inverse peak at 210-222 nm decreased upon mixing with $0.5 \mathrm{mM} \mathrm{Ca}{ }^{2+}$, which revealed that the content of the $\alpha$-helix structure of lipid-bound $\alpha$-Syn decreased. This result confirmed that the inhibitory effect of $0.5 \mathrm{mM} \mathrm{Ca}^{2+}$ on negatively charged vesicle clustering stemmed from the decreased amount of lipid-bound $\alpha$ Syn. When $10 \mathrm{mM} \mathrm{Ca}^{2+}$ was applied to the lipid-bound $\alpha$ Syn, the characteristic inverse peaks at 210-222 nm disappeared, and characteristic minima of random coil structures were observed at approximately $200 \mathrm{~nm}$, which implies the nearly complete removal of lipid-bound $\alpha$-Syn from the negatively charged lipid vesicles. The reason the restored peaks corresponding to a random coil shifted 

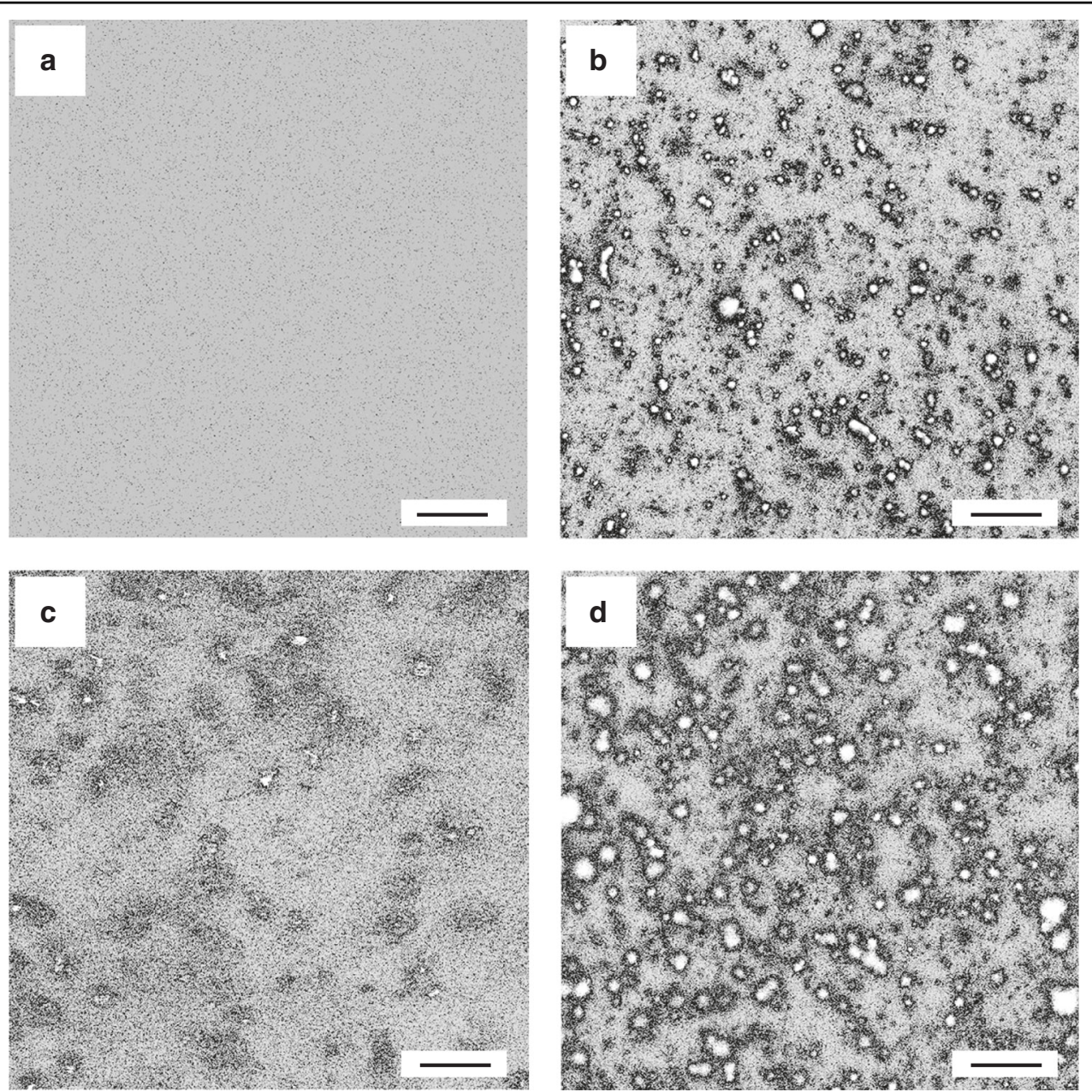

Fig. 4 Vesicle-clustering images of $12 \%$ PS vesicles under varied conditions. a Vesicles only, $\mathbf{b}$ with $\mathbf{a}-\mathrm{Syn}$ only, $\mathbf{c}$ with $\mathrm{a}-\mathrm{S} y \mathrm{n}$ and $0.5 \mathrm{mM} \mathrm{Ca}{ }^{2+}$, and $\mathbf{d}$ with a-Syn and $10 \mathrm{mM} \mathrm{Ca}^{2+}$. Scale bars: $10 \mu \mathrm{m}$

slightly compared with that of free $\alpha$-Syn is probably that a large amount of $\mathrm{Ca}^{2+}$ bound to the removed $\alpha$-Syn. Our $\mathrm{CD}$ spectrum experiments thus suggest that $\mathrm{Ca}^{2+}$, regardless of its concentration, inhibits the interaction between $\alpha$-Syn and negatively charged lipid membranes.

Speculating that the presence of $\mathrm{Ca}^{2+}$ affected $\alpha$-Syn's interaction with lipid membranes, and to confirm the interaction between $\alpha$-Syn and lipid vesicles, we performed molecular dynamics (MD) simulations to study the interaction of $\alpha$-Syn and lipid membranes mediated by $\mathrm{Ca}^{2+}$. The MD simulation method is a powerful approach to study MD and interactions. Previous studies have used MD simulations to study the conformations of $\alpha$-Syn in membranes ${ }^{28}$, the dynamics of $\mathrm{N}$-terminally acetylated $\alpha$ Syn with lipids ${ }^{29}$, the influence of membrane curvature on $\alpha$-Syn conformation ${ }^{30}$, and the effect of $\mathrm{Ca}^{2+}$ on different types of protein structures ${ }^{31}$. In particular, we tested the binding of $\alpha$-Syn's $\mathrm{N}$-terminal domain (amino acids $1-60$ ) to the anionic membrane in different concentrations of $\mathrm{Ca}^{2+}$. As shown in Fig. 7a, without $\mathrm{Ca}^{2+}$, the $\mathrm{N}$ terminus of $\alpha$-Syn quickly bound to the membrane with negatively charged lipids, consistent with previous findings that $\alpha$ Syn prefers to bind to anionic lipids via electrostatic interactions ${ }^{32,33}$. Snapshots of the details of $\alpha$-Syn's Nterminal binding to the membrane in the absence of $\mathrm{Ca}^{2+}$ can be found in Fig. S1. By contrast, when $\mathrm{Ca}^{2+}$ at concentrations of $2 \mathrm{mM}$ and $10 \mathrm{mM}$ was in the solution, the $\mathrm{N}$ terminus of $\alpha$-Syn had no contact with the membrane, as shown in Fig. 7b-e, depicting the distance evolutions calculated and the average values of $\alpha$-Syn's $N$ terminus to the lipid membrane, respectively, which confirmed the results suggesting that, without $\mathrm{Ca}^{2+}, \alpha$-Syn's $\mathrm{N}$ terminus is in close contact with the membrane. However, with $\mathrm{Ca}^{2+}$ at concentrations of 2 and $10 \mathrm{mM}, \alpha$-Syn's $\mathrm{N}$ terminus clearly distances itself from the membrane's surface. Figure $7 \mathrm{f}$, $\mathrm{g}$ shows that $\mathrm{H}$ bonds and contacts formed 

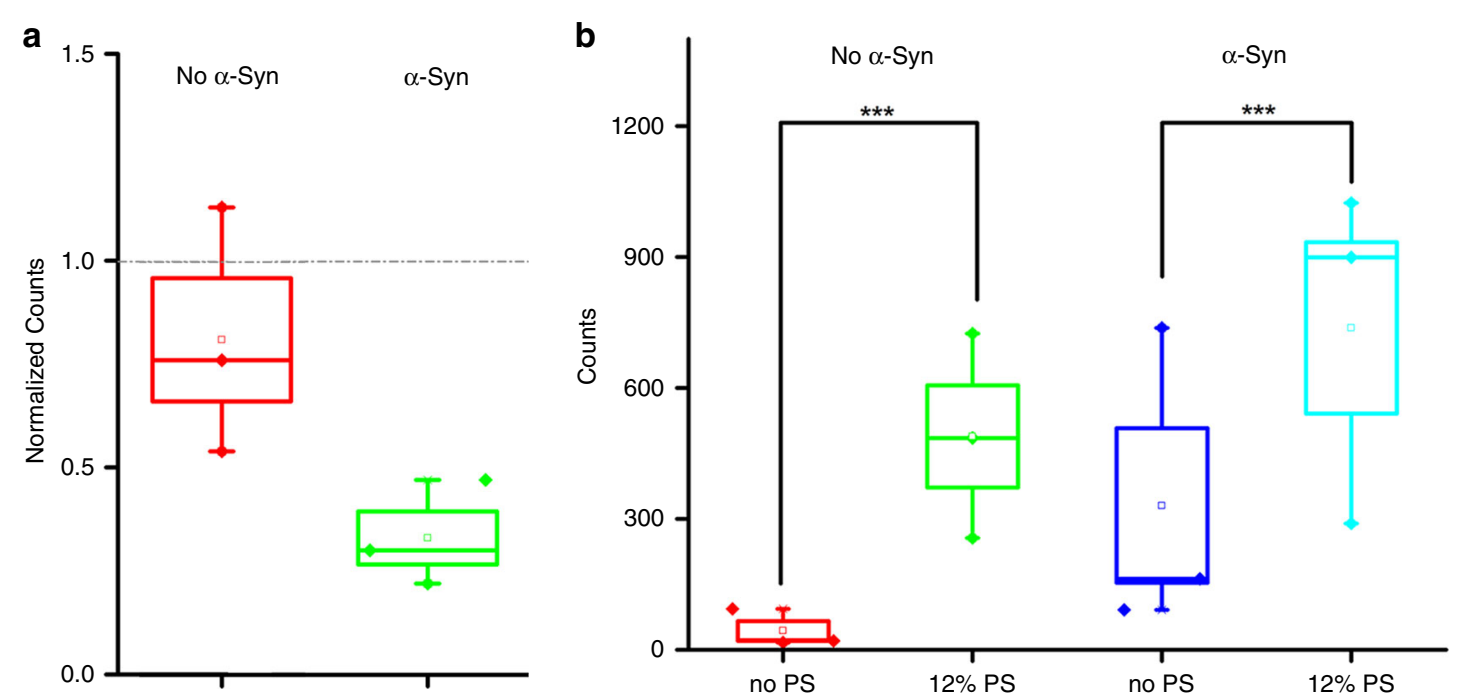

Fig. 5 The effect of charged lipids (PS) on the clustering counts of lipid vesicles at $\mathbf{a} 0.5 \mathrm{mM}$ and $\mathbf{b} 10 \mathrm{mM} \mathrm{Ca}{ }^{2+}$. In $\mathbf{a}$, the change is normalized to the number before adding $0.5 \mathrm{mM} \mathrm{Ca}^{2+}$

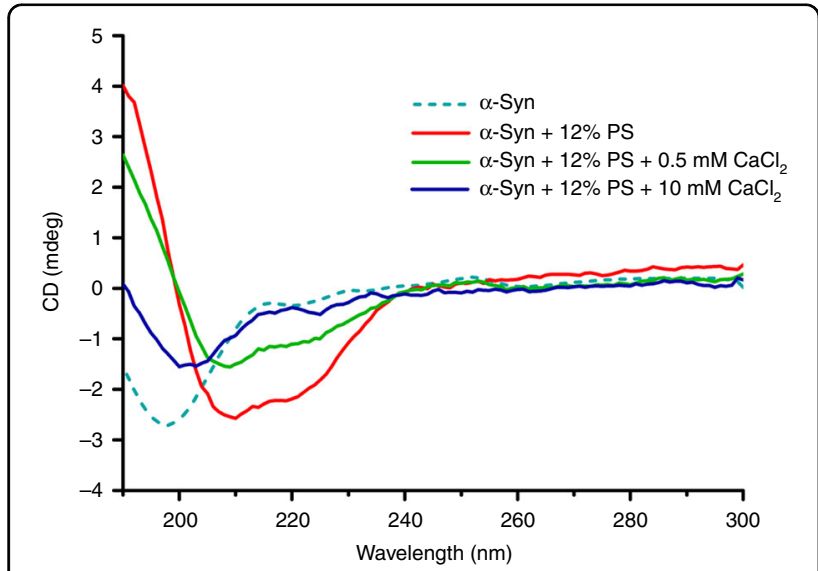

Fig. 6 The CD spectra of a-Syn after mixing with $12 \%$ PS vesicles and varied $\mathrm{Ca}^{2+}$ concentrations. The group with only wild-type aSyn was set as a control

between $\alpha$-Syn's $\mathrm{N}$ terminus and lipid membranes in the absence of $\mathrm{Ca}^{2+}$, whereas none of them formed in the presence of $\mathrm{Ca}^{2+}$. Figure 7h, i illustrates the interaction energies between $\alpha$-Syn's $\mathrm{N}$ terminus and lipid membranes, confirming that electrostatic interaction is the primary driving force of their binding, and that $\mathrm{Ca}^{2+}$ that competitively bound to negatively charged lipids inhibits the interaction between $\alpha$-Syn and the membranes. Consistent with the CD experiments (Fig. 6) and previous findings ${ }^{16,17}$, the MD results showed that $\mathrm{Ca}^{2+}$ binds to membranes with negatively charged lipids that block $\alpha$ Syn's binding to the membranes.

\section{Discussion}

$\alpha$-Syn, an intrinsically disordered protein in aqueous solution, is abundantly expressed throughout the central nervous system. Recent studies have suggested that $\alpha$-Syn binds to small synaptic vesicles with highly curved membranes via a conserved lipid-binding domain, senses and regulates the curvature of the membranes, and may be involved in both synaptic vesicle trafficking and the exo- and endocytosis of synaptic vesicles ${ }^{9-11}$. Furthermore, other research has proposed that $\alpha$-Syn can act as a bridge between membranes in which two helices bind to two synaptic vesicles and, in turn, cause vesicles to clus$\operatorname{ter}^{15}$. Similarly, another recent study showed that $\alpha$-Syn amyloid oligomers, acting as multivalent nanoparticles, can cause close contact and even hemifusion in negatively charged vesicles ${ }^{19}$. Because researchers have also proposed that $\mathrm{Ca}^{2+}$ can regulate the interaction between $\alpha$ Syn and lipid membranes ${ }^{16-18}$, we performed singlevesicle imaging to study vesicle clustering mediated by $\alpha$-Syn and the regulation of $\mathrm{Ca}^{2+}$.

Our results clearly indicate that $\alpha$-Syn can act as an intermediate in vesicle clustering by binding to negatively charged lipid vesicles, as shown in Fig. 2. A single-vesicle clustering assay revealed that the effect of $\mathrm{Ca}^{2+}$ in vesicle clustering with $\alpha$-Syn varies by concentration; at low concentrations, $\mathrm{Ca}^{2+}$ inhibited vesicle clustering with $\alpha$ Syn (Fig. 3), which can be attributed to the mechanism by 


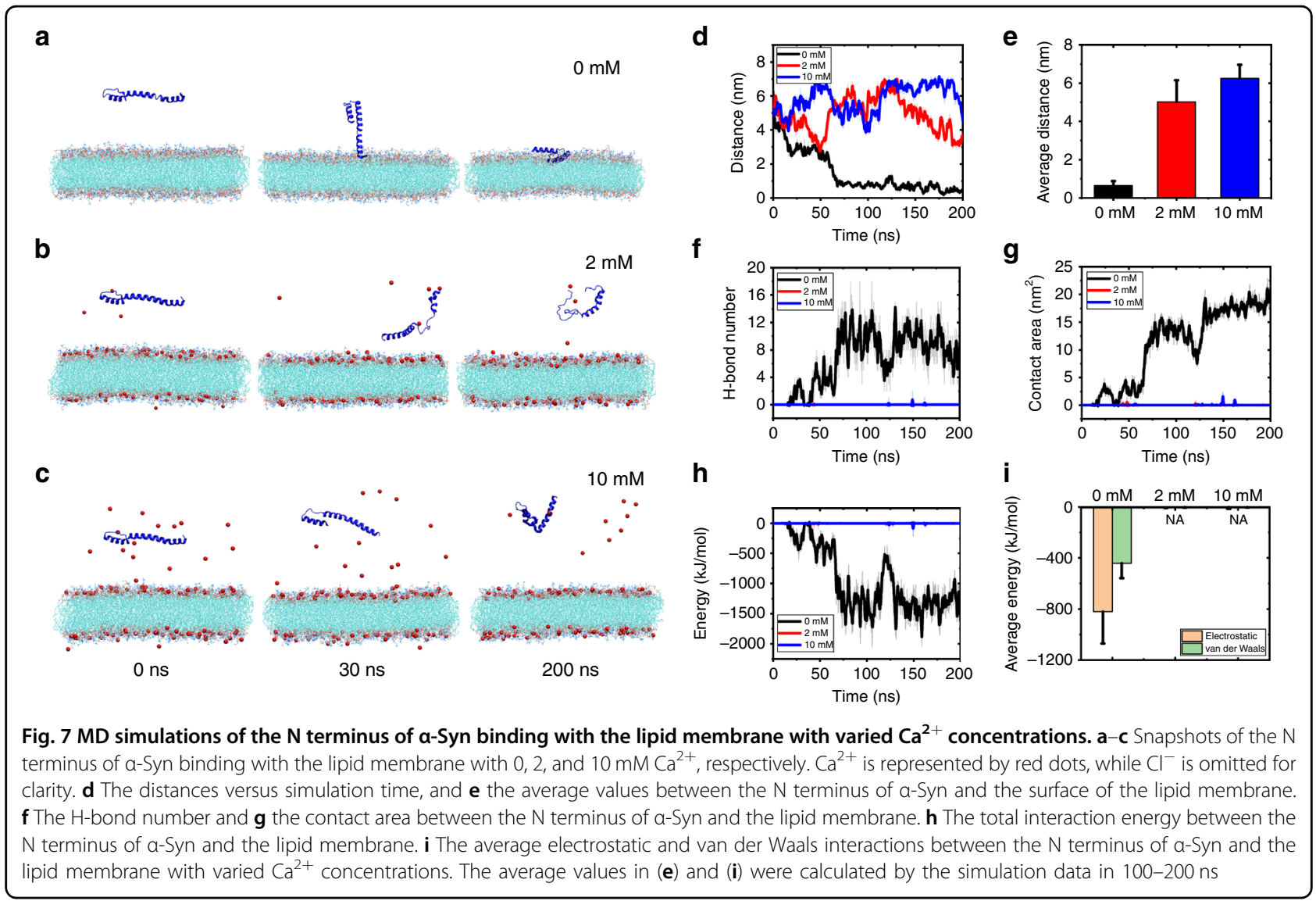

which the binding of $\mathrm{Ca}^{2+}$ to lipids blocks the interaction of $\alpha$-Syn with vesicles, such that $\alpha$-Syn cannot mediate vesicle clustering. This result is consistent with Zhang et al.'s ${ }^{16,17}$ finding that $\mathrm{Ca}^{2+}$ competitively binds to anionic lipids and thereby induces the dissociation of $\alpha$ Syn from membranes. At the same time, with more $\mathrm{Ca}^{2+}$ in the solution-that is, at high concentrations of $\mathrm{Ca}^{2+}-$ $\mathrm{Ca}^{2+}$ promotes vesicle clustering. $\mathrm{Ca}^{2+}$ without $\alpha$-Syn also enhanced vesicle clustering at high concentrations of $\mathrm{Ca}^{2+}$ (Fig. 5). However, CD spectroscopy experiments and MD simulations indicated that $\mathrm{Ca}^{2+}$ inhibited the interaction of $\alpha$-Syn's $\mathrm{N}$ terminus with vesicles regardless of the $\mathrm{Ca}^{2+}$ concentration (Figs. 6 and 7). The promotional role of high concentrations of $\mathrm{Ca}^{2+}$ in vesicle clustering may occur via the electrostatic interaction of $\mathrm{Ca}^{2+}$ and negatively charged lipids, which would corroborate previous findings that multivalent ions can cause ion bridges and tight coupling between lipid membranes ${ }^{27}$.

Our results also suggest that the mechanism of $\mathrm{Ca}^{2+}$ in regulating vesicle clustering varies with the $\mathrm{Ca}^{2+}$ concentration. At low concentrations, $\mathrm{Ca}^{2+}$ competes with $\alpha-$ Syn to bind to anionic lipids, which causes $\alpha$-Syn to dissociate from membranes such that vesicle clustering mediated by $\alpha$-Syn does not occur. By contrast, although $\mathrm{Ca}^{2+}$ may inhibit $\alpha$-Syn's interaction with membranes, a high concentration of $\mathrm{Ca}^{2+}$ can supply enough multivalent ions to drive vesicles into close contact and, in turn, form clusters.

\section{Conclusion}

$\alpha$-Syn has been shown to interact with isolated synaptic vesicles via its vesicle-binding domain, which results in the clustering of synaptic vesicles, and $\mathrm{Ca}^{2+}$ can regulate vesicle clustering mediated by $\alpha$-Syn. Using single-vesicle imaging, we studied how $\mathrm{Ca}^{2+}$ regulates vesicle clustering mediated by $\alpha$-Syn and the possible underlying mechanism. We showed not only that $\mathrm{Ca}^{2+}$ plays a critical role in vesicle clustering induced by $\alpha$-Syn, but also that different concentrations of $\mathrm{Ca}^{2+}$ exerted different effects on vesicle clustering mediated by $\alpha$-Syn via different mechanisms. Whereas a high concentration of $\mathrm{Ca}^{2+}$ promoted vesicle clustering in an $\alpha$-Syn-independent manner via the electrostatic interaction of $\mathrm{Ca}^{2+}$ and negatively charged lipids, low concentrations of $\mathrm{Ca}^{2+}$ inhibited vesicle clustering in an $\alpha$-Syn-dependent manner, in which $\mathrm{Ca}^{2+}$ competitively bound to anionic lipids, blocking $\alpha$-Syn's interaction with lipid membranes. Our bulk clustering experiments also confirmed the different effects of low and high concentrations of $\mathrm{Ca}^{2+}$ on vesicle clustering mediated by $\alpha$ Syn, while our CD experiments and MD simulations 
confirmed the underlying mechanisms, namely, that $\mathrm{Ca}^{2+}$ removes the $\mathrm{N}$ terminus of $\alpha$-Syn from the anionic membrane and inhibits vesicle clustering mediated by $\alpha$ Syn. Our work suggests that $\mathrm{Ca}^{2+}$ and negatively charged lipids play critical roles in vesicle clustering, and provides new insights into how $\alpha$-Syn regulates synaptic vesicle trafficking and synaptic transmission.

\section{Methods}

\section{Preparation of PEGylated slides for single-vesicle fluorescence imaging}

To study vesicle clustering, samples were loaded into a chamber assembled from quartz slides and glass coverslips. It is essential to have clean surfaces for single-vesicle fluorescence imaging. First, the slides and coverslips were rinsed and sonicated using Milli-Q $\mathrm{H}_{2} \mathrm{O}$, acetone, $1 \mathrm{M}$ $\mathrm{KOH}$ solution, and methanol, sequentially. After that, the slide and coverslip surfaces were burned for more than $1 \mathrm{~min}$ and $2 \mathrm{~s}$, respectively, to remove potential residues. To prevent nonspecific adsorption, the surfaces were PEGylated to achieve passivation, which included three steps, i.e., silanization, biotinylation, and washing. In brief, the slides and coverslips with clean surfaces were first incubated in amino silane ((3-(2-aminoethylamino) propyl) trimethoxysilane) solution (100 $\mathrm{ml}$ of methanol, $5 \mathrm{ml}$ of acetic acid, and $1 \mathrm{ml}$ of amino silane) for $10 \mathrm{~min}$, sonicated for $1 \mathrm{~min}$, and incubated for $10 \mathrm{~min}$ again. Each slide or coverslip was then blown dry from the edge using nitrogen gas after washing with Milli- $\mathrm{Q} \mathrm{H}_{2} \mathrm{O}$ followed by methanol. Finally, $100 \mu \mathrm{l}$ of reaction solution $(120 \mathrm{mg}$ of mPEG, $\sim 4 \mathrm{mg}$ of biotin-PEG, and $700 \mu \mathrm{l}$ of $0.1 \mathrm{M}$ sodium bicarbonate solution) was placed onto the imaging surface of the slide. Each slide was covered by one clean coverslip to be a set, which was incubated overnight and then rinsed with Milli-Q $\mathrm{H}_{2} \mathrm{O}$, dried, and stored at $-20^{\circ} \mathrm{C}$ for further use.

\section{Preparation of lipid vesicles}

We used two types of lipid vesicles with fluorescent dyes, i.e., DiI (DiIC18(3)) and DiD (DiIC18(5)), for singlevesicle clustering experiments. Before mixing the lipid reagents, the glass syringe was washed three times with chloroform. The lipid components were added into the glass tube wrapped with a piece of foil with a small hole at the top. For preparation of $12 \%$ PS vesicles, $103.8 \mu \mathrm{l}$ of DOPC (1,2-dioleoyl-sn-glycero-3-phosphocholine), $29.8 \mu \mathrm{l}$ of DOPE (1,2-dioleoyl-sn-glycero-3-phosphoethanolamine), $48.6 \mu \mathrm{l}$ of DOPS (1,2-dioleoyl-sn-glycero-3(phospho-L-serine)), 6.4 $\mu \mathrm{l}$ of Bio-PE (1,2-dioleoyl-snglycero-3-phosphoethanolamine-N-(biotinyl)) (only for DiI vesicles) and fluorescent dye (100 $\mu \mathrm{l}$ of DiI or DiD) were included in the composition required. The lipid composition of the no-PS vesicles was similar to that of $12 \%$ PS vesicles, except for the absence of DOPS. To form a thin lipid film, the mixture in the tube was subjected to vacuum in a ball container and rotated every $30 \mathrm{~min}$ until the organic solvent was completely evaporated. Subsequently, $500 \mu \mathrm{l}$ of HEPES buffer $(25 \mathrm{mM}$ HEPES and $100 \mathrm{mM} \mathrm{NaCl}, \mathrm{pH}$ 7.4) was added to hydrate the lipid film. Finally, the solution was frozen and thawed at least 5-6 times to obtain vesicles that were filtered through a membrane with $50-\mathrm{nm}$ pores. The diameter distribution of 40-50-nm liposomes was previously measured by $\mathrm{TEM}^{34}$. The dispersed vesicles were stored at $-80^{\circ} \mathrm{C}$ for further experiments.

\section{Single-vesicle clustering experiments}

The no-PS or $12 \%$ PS vesicles with DiI were first immobilized on the imaging surface of PEGylated quartz slides via interaction between biotin and NeutrAvidin. The imaging area should be fully covered by DiI vesicles and produced a homogeneous distribution. After incubation for $30 \mathrm{~min}$ and buffer change, wild-type $\alpha$-Syn was injected into the sample chamber and incubated for $15 \mathrm{~min}$. The channel without supply of $\alpha$-Syn was set as a control. The DiD vesicle solution corresponding to the immobilized DiI vesicles at the required protein-lipid ratio was injected into each channel after buffer exchange to remove unbound $\alpha$-Syn. To investigate the effects of $\mathrm{Ca}^{2+}$ or other divalent ions on vesicle clustering, $\mathrm{Ca}^{2+}$ or other divalent ions were mixed with $\mathrm{DiD}$ vesicles at different concentrations and injected into the sample chambers immediately. Before imaging under wide-field TIRFM, samples in the channels were incubated for $20 \mathrm{~min}$ and washed with HEPES buffer three times to remove uncombined vesicles.

DiI and $\mathrm{DiD}$ vesicles were detected and imaged after the dyes were excited by a green laser $(532 \mathrm{~nm})$ and a red laser $(633 \mathrm{~nm})$, respectively. The CCD camera and smCamera program were used to acquire and analyze the images, respectively. The number of vesicles clustered was determined by counting the $\mathrm{DiD}$ vesicles whose fluorescent spots were shown in the acceptor channel on each image $(45 \times 90 \mu \mathrm{m})$. Approximately, 15 random locations were imaged and analyzed for each sample channel. The compared results are expressed as the mean \pm standard deviation. One-way analysis of variance (ANOVA) with Tukey's test was used to determine the statistical significance among different groups. When $P<0.001$, the difference was considered extremely significant $\left({ }^{* * * *}\right)$.

\section{Bulk clustering}

To investigate bulk vesicle clustering, the DiD vesicles were dispersed and diluted in $3 \mathrm{wt} \%$ mPEG (Mw: 5000) solution to $2 \mu \mathrm{M}$. The 1:500 protein-lipid ratio was used to study the bulk clustering in the presence of $4 \mathrm{nM} \alpha$ Syn. A $10 \mathrm{mM}$ or $0.5 \mathrm{mM} \mathrm{Ca}^{2+}$ solution was added to the mixture of $\alpha$-Syn and lipid vesicles to observe the effect of 
$\mathrm{Ca}^{2+}$. The mixture was incubated for more than $20 \mathrm{~min}$ before imaging the samples excited by red laser light with confocal microscopy. Imaging was performed using 35$\mathrm{mm}$ glass-bottom dishes (MatTek).

\section{CD spectroscopy}

The lipid composition of the $12 \%$ PS vesicles used for the CD experiments includes $43 \mathrm{~mol} \%$ DOPC, $45 \mathrm{~mol} \%$ DOPE, and $12 \mathrm{~mol} \%$ DOPS. Dried lipid films were rehydrated in deionized water to a concentration of $5 \mathrm{mM}$. Vesicles were formed after at least six freeze (liquid $\mathrm{N}_{2}$ )-thaw $\left(37^{\circ} \mathrm{C}\right.$ water bath) cycles. Before use, the vesicles were sonicated three times in low-power mode. Each sonication was performed for $5 \mathrm{~min}$. $\alpha$-Syn was dissolved in Tris- $\mathrm{HCl}$ buffer $(25 \mathrm{mM}$ Tris- $\mathrm{HCl}$ and $10 \mathrm{mM} \mathrm{NaCl}$, $\mathrm{pH}$ 7.4) to form a protein solution of $2.5 \mathrm{mg} / \mathrm{ml}$, which was dialyzed in $300 \mathrm{ml}$ of CD buffer overnight before use. The final protein solution for detection was $10 \mu \mathrm{M}$. The protein-to-lipid ratio in the mixture of $\alpha$-Syn and lipids used for each test was $1: 100 . \mathrm{CaCl}_{2}(0.5 \mathrm{mM}$ or $10 \mathrm{mM})$ dissolved in Tris- $\mathrm{HCl}$ buffer was mixed with the corresponding lipid-bound protein solution. The secondary structure of lipid-free or lipid-bound $\alpha$-Syn was determined by CD spectroscopy (Aviv 215 CD spectrophotometer). Wavelength scans were taken in $0.5-\mathrm{nm}$ steps with a 3-s averaging time and 0.333-s settling time. Spectra were collected from $300 \mathrm{~nm}$ to $190 \mathrm{~nm}$ at $25^{\circ} \mathrm{C}$ in a $0.1-\mathrm{mm}$ path-length quartz cuvette containing samples. The baseline was corrected by the blank (buffer only). Each spectrum was averaged from three repeats. The corrected signal data of each $\mathrm{CD}$ spectrum were smoothed with the adjacent-averaging method and then plotted using Origin8.5.

\section{Preparation of MD simulation models}

The lipid bilayers were generated by the Membrane Builder online service CHARMM-GUI ${ }^{35,36}$. Here, the lipid composition was adopted as DOPC:DOPE:DOPS with a ratio of $45 \%: 43 \%: 12 \%$. DOPC, DOPE, and DOPS are abbreviations for 1,2-dioleoyl-sn-glycero-3-phosphocholine, 1,2-dioleoyl-sn-glycero-3-phosphoethanolamine, and 1,2-dioleoyl-sn-glycero-3-phospho-L-serine, respectively. To reduce the computational demand, the membrane was built as a flat membrane. To mimic the packing density of lipids in a curved membrane, we applied tension to the flat membrane by $\sigma=\left\langle L_{z} / 2\left[p_{z z}-1 / 2\left(p_{x x}+p_{y y}\right)\right]\right\rangle$, where $\sigma$ is the membrane tension, $p_{i j}$ is the $i j$ component of the pressure, $L_{z}$ is the box length along the $z$ direction, and $<\cdot>$ represents the time average $e^{37-39}$. According to the packing density of lipids in vesicles (e.g., $~ 1.19-1.35$ lipids per square nanometer for a vesicle with an $\sim 20$-nm diameter ${ }^{40,41}$ ), we applied $p_{z z}=1$ bar, $p_{x x}=p_{y y}=-28$ bar for $15 \mathrm{~ns}$ to achieve a packing density of $\sim 1.28$ lipids per square nanometer of the membrane, as shown in Fig. S2.
The system was solvated in an $\sim 15 \times 15 \times 12 \mathrm{~nm}^{3}$ TIP3P ${ }^{42}$ water box, with $\sim 65,000$ water molecules and an appropriate number of sodium ions added to neutralize the system.

According to a previous study, $\mathrm{Ca}^{2+}$ in solution will significantly bind to the membrane with negatively charged lipids ${ }^{27,43}$. To prepare the membrane model with $2 \mathrm{mM}$ and $10 \mathrm{mM} \mathrm{Ca}{ }^{2+}$, we gradually added $\mathrm{CaCl}_{2}$ molecules to the solution, and $\mathrm{Ca}^{2+}$ bound to the membrane, as shown in Fig. S3. Until the concentration of $\mathrm{Ca}^{2+}$ in solution was static at $2 \mathrm{mM}$ and $10 \mathrm{mM}$ for $20 \mathrm{~ns}$, the structures were extracted for simulations of $\alpha$-Syn binding, as shown in Fig. S3. The structure of $\alpha$-Syn was adopted from PDB: $1 \mathrm{XQ}^{13}$. To reduce the computational demand, only the $\mathrm{N}$ terminus of $\alpha$-Syn (i.e., residues $1-60)$ was used in the simulations.

\section{MD simulation method}

All of the simulations were performed using the GROMACS package ${ }^{44}$ with the CHARMM36 force field ${ }^{45}$. Periodic boundary conditions were applied, and the temperature was coupled to $310.25 \mathrm{~K}$ with the V-rescale algorithm ${ }^{46}$. To maintain the packing density of lipids, the pressure was coupled by the Parrinello-Rahman method as $p_{z z}=1$ bar, $p_{x x}=p_{y y}=-28$ to apply membrane tension ${ }^{47}$. The LINCS algorithm was applied to constrain the covalent bonds with $\mathrm{H}$ atoms ${ }^{48}$. The time step of the simulations was $2.0 \mathrm{fs}$. The particle mesh Ewald method was used to calculate the long-range electrostatic interactions $^{49}$. The cutoff of the nonbonded interactions was set to $12 \AA$. All graphics and visualization analyses were processed using the VMD package ${ }^{50}$.

\section{Acknowledgements}

We thank Dr. Chirlmin Joo for help in preparing the figure of the TIRFM setup and Dr. Tom Thompson for help with the CD experiments. B.B., D.L., L.Z., W.D., and B.J. were supported by funds from the National Natural Science Foundation of China (NSFC 11932017, 11772054, 11772055, 11532009, 11902051, and 31871031). W.D. was supported by the Sichuan Science and Technology Program (2019YJ0481). D.L. was supported by the Fundamental Research Funds for the Central Universities (Grant no. 2019QNA4060). J.D. was supported by the Michael J Fox Foundation (ID 16661).

\footnotetext{
Author details

${ }^{1}$ Department of Cancer Biology, University of Cincinnati College of Medicine, Cincinnati, $\mathrm{OH} 45267$, USA. ${ }^{2}$ Biomechanics and Biomaterials Laboratory, Department of Applied Mechanics, Beijing Institute of Technology, Beijing 100081, China. ${ }^{3}$ Key Laboratory of Medical Electrophysiology of Ministry of Education and Medical Electrophysiological Key Laboratory of Sichuan Province, Institute of Cardiovascular Research, Southwest Medical University, Luzhou, Sichuan 646000, China. ${ }^{4}$ Institute of Biomedical Engineering and Health Sciences, Changzhou University, Changzhou, Jiangsu 213164, China. ${ }^{5}$ Institute of Applied Mechanics, Department of Engineering Mechanics, Zhejiang University, Hangzhou 310027, China. Key Laboratory for Nonequilibrium Synthesis and Modulation of Condensed Matter (Ministry of Education), School of Science, Xi'an Jiaotong University, Xi'an 710049, China. ${ }^{7}$ Beijing Advanced Innovation Center for Biomedical Engineering, Beijing 100191, China
} 


\section{Author contributions}

D.L. and J.D. designed and directed the study. B.C. and Y.Z. performed singlevesicle and CD experiments. J.L., X.X., and B.B. conducted MD simulation. L.Z. carried TEM measurements. D.L., W.D., B.J., and J.D. wrote the manuscript.

\section{Conflict of interest}

The authors declare that they have no conflict of interest.

Supplementary information accompanies this paper at https://doi.org/ 10.1038/s41378-020-0147-1.

Received: 23 December 2019 Revised: 18 January 2020 Accepted: 3 February 2020

Published online: 29 June 2020

\section{References}

1. Goedert, M. Alpha-synuclein and neurodegenerative diseases. Nat. Rev. Neurosci. 2, 492-501 (2001).

2. Spillantini, M. G. et al. Alpha-synuclein in Lewy bodies. Nature $\mathbf{3 8 8}, \mathbf{8 3 9 - 8 4 0}$ (1997).

3. Iyer, A. \& Claessens, M. M. A. E. Disruptive membrane interactions of alphasynuclein aggregates. Biochim. Biophys. Acta 1867, 468-482 (2019).

4. Goldberg, M. S. \& Lansbury, P. T. Is there a cause-and-effect relationship between alpha-synuclein fibrillization and Parkinson's disease? Nat. Cell Biol. 2, E115-E119 (2000).

5. Bucciantini, M. et al. Inherent toxicity of aggregates implies a common mechanism for protein misfolding diseases. Nature 416, 507-511 (2002).

6. Bu, B. et al. N-terminal acetylation preserves alpha-synuclein from oligomerization by blocking intermolecular hydrogen bonds. ACS Chem. Neurosci. 8, 2145-2151 (2017)

7. Hu, R. et al. Intrinsic and membrane-facilitated alpha-synuclein oligomerization revealed by label-free detection through solid-state nanopores. Sci. Rep. $\mathbf{6}$, 20776 (2016).

8. Li, X. et al. Label-free detection of early oligomerization of alpha-synuclein and its mutants A30P/E46K through solid-state nanopores. Nanoscale 11, 6480-6488 (2019).

9. Wang, C. C. et al. Versatile structures of alpha-synuclein. Front. Mol. Neurosci. $\mathbf{9}$, 48 (2016).

10. Diao, J. J. et al. Native alpha-synuclein induces clustering of synaptic-vesicle mimics via binding to phospholipids and synaptobrevin-2NAMP2. Elife 2, e00592 (2013).

11. Lautenschlager, J., Kaminski, C. F. \& Schierle, G. S. K. Alpha-synuclein-regulator of exocytosis, endocytosis, or both? Trends Cell Biol. 27, 468-479 (2017).

12. Burre, J. et al. Properties of native brain alpha-synuclein. Nature 498, E4-E6 (2013). discussion E6-7.

13. Ulmer, T. S. et al. Structure and dynamics of micelle-bound human alphasynuclein. J. Biol. Chem. 280, 9595-9603 (2005).

14. Georgieva, E. R. et al. Membrane-bound alpha-synuclein forms an extended helix: Long-distance pulsed ESR measurements using vesicles, bicelles, and rodlike micelles. J. Am. Chem. Soc. 130, 12856 (2008).

15. Dikiy, I. \& Eliezer, D. Folding and misfolding of alpha-synuclein on membranes. Biochim. Biophys. Acta 1818, 1013-1018 (2012).

16. Zhang, Z. T. et al. Ca2+ modulating alpha-synuclein membrane transient interactions revealed by solution NMR spectroscopy. Biochim. Biophys. Acta 1838, 853-858 (2014).

17. Zhang, Z. T. et al. Calcium accelerates SNARE-mediated lipid mixing through modulating alpha-synuclein membrane interaction. Biochim. Biophys. Acta 1860, 1848-1853 (2018).

18. Lautenschlager, J. et al. C-terminal calcium binding of alpha-synuclein modulates synaptic vesicle interaction. Nat. Commun. 9, 712 (2018).

19. Stefanovic, A. N. D. et al. Alpha-synuclein amyloid oligomers act as multivalent nanoparticles to cause hemifusion in negatively charged vesicles. Small 11, 2257-2262 (2015).

20. Tian, Z. Q. et al. Biochemical studies of membrane fusion at the single-particle level. Prog. Lipid Res. 73, 92-100 (2019).

21. Fish, K. N. Total internal reflection fluorescence (TIRF) microscopy. Current protocols in cytometry, 2009. Chapter 12: p. Unit 12.18.
22. Fiolka, R. P. \& Stemmer, A. Extending the resolution in total internal reflection fluorescence (TIRF) microscopy. Biophys. J. 96, 636a (2009).

23. Ma, D. F. et al. Detecting single-molecule dynamics on lipid membranes with quenchers-in-a-liposome FRET. Angew. Chem. Int. Ed. Engl. 58, 5577-5581 (2019).

24. Gong, J. et al. C-terminal domain of mammalian complexin-1 localizes to highly curved membranes. Proc. Natl Acad. Sci. USA 113, E7590-E7599 (2016).

25. Lai, Y. et al. Nonaggregated alpha-synuclein influences SNAREdependent vesicle docking via membrane binding. Biochemistry 53, 3889-3896 (2014).

26. Cai, B. et al. Single-vesicle measurement of protein-induced membrane tethering. Colloids Surf. B Biointerfaces 177, 267-273 (2019).

27. Fink, L. et al. Structure and interactions between charged lipid membranes in the presence of multivalent ions. Langmuir 35, 9694-9703 (2019).

28. Vermaas, J. V. \& Tajkhorshid, E. Conformational heterogeneity of alpha-synuclein in membrane. Biochim. Biophys. Acta $\mathbf{1 8 3 8}$ 3107-3117 (2014).

29. Rossetti, G. et al. Conformational ensemble of human alpha-synuclein physiological form predicted by molecular simulations. Phys. Chem. Chem. Phys. 18, 5702-5706 (2016).

30. Perlmutter, J. D., Braun, A. R. \& Sachs, J. N. Curvature dynamics of alphasynuclein familial Parkinson disease mutants: molecular simulations of the micelle- and bilayer-bound forms. J. Biol. Chem. 284, 7177-7189 (2009)

31. Chen, G. et al. Plasticizing silk protein for on-skin stretchable electrodes. Adv. Mater. 30, e1800129 (2018).

32. Galvagnion, C. et al. Chemical properties of lipids strongly affect the kinetics of the membrane-induced aggregation of alpha-synuclein. Proc. Natl Acad. Sci. USA 113, 7065-7070 (2016).

33. Zhu, M. \& Fink, A. L. Lipid binding inhibits alpha-synuclein fibril formation. J. Biol. Chem. 278, 16873-16877 (2003).

34. Hu, Y. et al. SNARE-reconstituted liposomes as controllable zeptoliter nanoreactors for macromolecules. Adv. Biosyst. 1, 1600018 (2017).

35. Lee, J. et al. CHARMM-GUI input generator for NAMD, GROMACS, AMBER, OpenMM, and CHARMM/OpenMM simulations using the CHARMM36 additive force field. J. Chem. Theory Comput. 12, 405-413 (2016).

36. Jo, S. et al. CHARMM-GUI Membrane Builder for mixed bilayers and its application to yeast membranes. Biophys. J. 97, 50-58 (2009).

37. Leontiadou, H., Mark, A. E. \& Marrink, S. J. Molecular dynamics simulations of hydrophilic pores in lipid bilayers. Biophys. J. 86, 2156-2164 (2004).

38. Bu, B. et al. Cholesterol suppresses membrane leakage by decreasing water penetrability. Soft Matter 14, 5277-5282 (2018).

39. Wang, Q. C. et al. Heterogeneous oxidization of graphene nanosheets damages membrane. Sci. China Phys. Mech. Astron. 62, 064611 (2019).

40. Cornell, B. A., Middlehurst, J. \& Separovic, F. The molecular packing and stability within highly curved phospholipid-bilayers. Biochim. Biophys. Acta 598, 405-410 (1980).

41. Huang, C. \& Mason, J. T. Geometric packing constraints in egg phosphatidylcholine vesicles. Proc. Natl Acad. Sci. USA 75, 308-310 (1978).

42. Jorgensen, W. L. et al. Comparison of simple potential functions for simulating liquid water. J. Chem. Phys. 79, 926 (1983).

43. Hauser, H. \& Shipley, G. G. Interactions of divalent-cations with phosphatidylserine bilayer-membranes. Biochemistry 23, 34-41 (1984).

44. Van Der Spoel, D. et al. GROMACS: fast, flexible, and free. J. Comput. Chem. 26 1701-18. (2005)

45. Klauda, J. B. et al. Update of the CHARMM all-atom additive force field for lipids: validation on six lipid types. J. Phys. Chem. B 114, 7830-43. (2010).

46. Bussi, G., Donadio, D. \& Parrinello, M. Canonical sampling through velocity rescaling. J. Chem. Phys. 126, 014101 (2008)

47. Parrinello, M. \& Rahman, A. Polymorphic transitions in single-crystals-a new molecular-dynamics method. J. Appl. Phys. 52, 7182-7190 (1981).

48. Hess, B. P-LINCS: a parallel linear constraint solver for molecular simulation. J. Chem. Theory Comput. 4, 116-122 (2008).

49. Essmann, U. et al. A smooth particle mesh Ewald method. J. Chem. Phys. 103 8577-8593 (1995)

50. Humphrey, W., Dalke, A. \& Schulten, K. VMD: visual molecular dynamics. J. Mol Graph. 14, 33-38 (1996). 